\title{
From a hard nuclear state towards a soft nuclear repository state - participation, co-designing, learning and reversibility in the site selection process for a nuclear waste repository
}

\author{
Achim Brunnengräber, Maria Rosaria Di Nucci, Lucas Schwarz, and Dörte Themann \\ Forschungszentrum für Umweltpolitik, Freie Universität Berlin, Berlin, 14195, Germany
}

Correspondence: Achim Brunnengräber (achim.brunnengraeber@fu-berlin.de)

Published: 10 November 2021

\begin{abstract}
Since 2013 the site search for a repository for highly radioactive waste has been taking place in Germany within the framework of a new governance architecture and under new political guidelines. Based on experiences with nuclear politics in the past, Jungk (1977) coined the term hard nuclear state, characterized by decisions made in a top-down manner. The Decide-Announce-Defend (DAD) strategy, which branded the nuclear state at that time, led to conflicts, mistrust of authorities and blockages. In particular, massive resistance developed against the planned final repository site at Gorleben.

Nowadays, after more than 60 years deploying nuclear energy, the (energy) political balance of power has fundamentally changed. Parts of the anti-nuclear movement have been integrated into the political party system and have contributed significantly to the nuclear phase-out. In the course of this, the unfinished task of final disposal has been readdressed: with the Repository Site Selection Act (StandAG, 2017), which was passed in 2013 and amended in 2017, an ongoing process of public participation is stated. The site selection process is required to be learning, self-questioning, science-based, reversible, and participatory. The StandAG $\S 5$ not only provides a basis for a fundamental dialogue between the regulator, the operator, and the public, but also for „co-design“ by common citizens.

The StandAG considers various elements from different participation-friendly theories of democracy as well as specific governance concepts, which we refer to collectively as the soft nuclear repository state (cf. Brunnengräber, 2021). Its characteristics need to be worked out, as the StandAG only provides some indications, but no criteria, for what good and sufficient participation in the site selection process means and what its conditions for success should be. Based on preliminary considerations on democratic theory and governance aspects (part 1), we present what good participation could mean in the current procedure and what the framework conditions for good participation could be (part 2). Additionally, we present main findings from participatory observations from the ongoing site search process and identify conditions and indications of a successful future participation process based on the ongoing process (part 3). In the résumé, we turn to the question of which of the democracy-theoretical elements of the soft repository state are already recognizable in the present procedure, but also whether the current procedure provides additional indications towards the soft nuclear repository state (part 4).
\end{abstract}

Kurzfassung. Die Standortsuche für ein Endlager für hochradioaktive Abfälle findet in Deutschland seit 2013 im Rahmen einer neuen Governance-Architektur und unter neuen politischen Vorgaben statt. Jungk (1977) prägte nach den Erfahrungen mit der Atompolitik den Begriff des harten Atomstaats, der Entscheidungen im Top-downVerfahren fällte. Die Decide-Announce-Defend (DAD)-Strategie, die den damaligen Atomstaat ganz wesentlich charakterisierte, führte zu Konflikten, Misstrauen gegenüber den Behörden und (auch gerichtlich erstrittenen) Blockaden. Insbesondere gegen den geplanten Endlagerstandort Gorleben hatte sich massiver Widerstand entwickelt. 
Nach über 60 Jahren Nutzung der Atomenergie haben sich die (energie)politischen Kräfteverhältnisse jedoch grundsätzlich verändert. Teile der Anti-Atom-Bewegung sind in das politische Parteiensystem integriert und haben wesentlich zum sog. Atomausstieg beigetragen. Im Zuge dessen wird die unerledigte Aufgabe der Endlagerung neu adressiert: Im 2013 verabschiedeten und 2017 novellierten Standortauswahlgesetz (StandAG, 2017) wird ein fortlaufender Prozess der Öffentlichkeitsbeteiligung festgeschrieben. Das Verfahren der Standortsuche soll lernend und selbsthinterfragend, wissenschaftsbasiert, reversibel und partizipativ sein. StandAG $\S 5$ sieht nicht nur einen grundlegenden Dialog zwischen Aufsichtsbehörde, Betreiber und Öffentlichkeit vor, sondern auch eine „Mitgestaltung“ durch Bürger*innen.

Das StandAG weist in diesem Sinne Elemente aus verschiedenen beteiligungsfreundlichen Demokratietheorien, sowie spezifischen Governance-Konzepten auf, die wir in der Summe als weichen Endlagerstaat bezeichnen (vgl. Brunnengräber, 2021). Dessen Charakteristika gilt es herauszuarbeiten, da das StandAG zwar einige Hinweise, jedoch keine Kriterien dafür gibt, was ,gute“ und ausreichende Beteiligung in der Standortauswahl bedeutet und was somit deren Gelingensbedingungen wären. Basierend auf Demokratietheorien und Governance Vorüberlegungen zu Governance (1. Teil) stellen wir vor, was gute Beteiligung im jetzigen Verfahren bedeuten könnte und was die Rahmenbedingungen guter Beteiligung wären (2. Teil). Ergänzend präsentieren wir Ergebnisse unserer teilnehmenden Beobachtungen aus dem laufenden Standortsuchverfahren und identifizieren Bedingungen und Hinweise auf Elemente eines erfolgreichen zukünftigen Beteiligungsprozesses auf der Grundlage des realen Prozesses (3. Teil). Im Resümee wenden wir uns der Frage zu, welche der demokratietheoretischen Elemente des weichen Endlagerstaats bereits im Verfahren erkennbar sind, sowie, ob das derzeitige Verfahren weitere Hinweise auf den weichen Endlagerstaat liefert (4. Teil).

Financial support. This research has been supported by the Bundesministerium für Wirtschaft und Energie and the Niedersächsisches Vorab der Volkswagenstiftung (grant no. 02E11849c).

\section{References}

Brunnengräber, A.: Vom starken zum weichen Atomstaat, in: Robuste Langzeit-Governance bei der Endlagersuche: Soziotechnische Herausforderungen im Umgang mit hochradioaktiven Abfällen, edited by: Brohmann, B., Brunnengräber, A., Hocke, P., and Isidoro Losada, A. M., transcript-Verlag, Bielefeld, 6178, https://doi.org/10.14361/9783839456682-005, 2021.
Jungk, R.: Der Atom-Staat: Vom Fortschritt in die Unmenschlichkeit, Rororo, 7288: rororo-Sachbuch, Rowohlt, Reinbek bei Hamburg, 182 pp., 1977.

StandAG: Gesetz zur Suche und Auswahl eines Standortes für ein Endlager für hochradioaktive Abfälle: Standortauswahlgesetz 1. Novelle, 2017. 